CLINICAL STUDY

\title{
Changes in cerebral blood flow velocity in patients with hypothyroidism
}

\author{
Uygar Utku, Mustafa Gokce and Mesut Özkaya \\ Department of Neurology, Medical Faculty, Kahramanmaras Sutcu Imam University, 46050 Kahramanmaras, Turkey \\ (Correspondence should be addressed to U Utku; Email: utkuzar@gmail.com)
}

\begin{abstract}
Background: At present, hypothyroidism is a well-known risk factor for cardiovascular disorders. The aim of this study was to assess the effects of hypothyroidism on cerebral blood flow velocity with transcranial Doppler (TCD) ultrasonography.

Design and methods: In this study, 30 subjects were enrolled for clinical, subclinical, and healthy control groups. Bilateral middle cerebral artery (MCA) peak-systolic, end-diastolic, and mean blood flow velocities; Gosling's pulsatility index values; and Pourcelot's resistance index values were recorded and compared with each other. TCD was performed in clinical hypothyroid patients after they became euthyroid with thyroid hormone replacement therapy (HRT). The initial and post-HRT results for the clinical hypothyroid group were then compared and evaluated.

Results: There were 30 subjects in each group. Men/women ratio and mean age in clinical hypothyroid, subclinical hypothyroid, and control groups were 3/27,4/26, and 5/25, and 37.4, 34.4, and 36.7 respectively. Peak-systolic, end-diastolic, and mean blood flow velocities of bilateral MCA were similar in clinical and subclinical hypothyroid groups but significantly higher when compared with the control group. After adequate thyroid HRT in clinical hypothyroid group, the peak-systolic, enddiastolic, and mean blood flow velocities were significantly decreased.

Conclusions: Increased cerebral blood flow velocities were observed in clinical and subclinical patients with hypothyroidism. The normalization of increased blood flow velocity with thyroid HRT suggests a reversible condition.
\end{abstract}

European Journal of Endocrinology 165 465-468

\section{Introduction}

Hypothyroidism modifies the risk factors of atherosclerosis, and indirectly affects atherogenesis and also has direct effects on the vasculature (1). Epidemiological studies have suggested that patients with hypothyroidism have accelerated coronary atherosclerosis. Hypercholesterolemia, hyperhomocysteinemia, increased C-reactive protein, hypertension, and impaired endothelial function in hypothyroid state enhance the atherogenesis $(2,3)$. The Rotterdam study showed a higher prevalence of myocardial infarction in women with subclinical hypothyroidism compared with euthyroid women (4). Nagasaki et al. (5) showed a decrease in carotid intima-media thickness in hypothyroid patients after normalization of thyroid function. There is some suspicion that atherosclerosis due to hypothyroidism may cause a stroke, but this condition is not yet clear $(6,7)$. Ojamaa et al. $(8,9)$ reported that thyroid hormone induced relaxation of vascular smooth muscle cells have a direct effect and hence increased arterial stiffness is seen in hypothyroidism. To date, there is no study about cerebral blood velocity change in hypothyroidism. As a consequence, we investigated whether cerebral blood flow velocities changed in hypothyroidism.

\section{Materials and methods}

\section{Subjects}

The study was conducted in the outpatient clinics of Endocrinology and Neurosonology Laboratory of our hospital. Newly diagnosed 30 patients with clinical hypothyroidism and 30 patients with subclinical hypothyroidism were included. Thirty healthy control subjects were also enrolled in the study. Age and gender of the control group were similar to the other groups. Diagnosis of clinical and subclinical hypothyroidism was made by endocrinology physicians according to physical findings of patients and laboratory levels of their thyroid hormones. Patients who had possible risks for atherosclerosis, such as diabetes, hypertension, and 
Table 1 Transcranial Doppler data of clinical hypothyroid group compared with control group.

\begin{tabular}{lccc}
\hline & $\begin{array}{c}\text { Clinical } \\
\text { hypothyroid }\end{array}$ & Control & $\boldsymbol{P}$ \\
\hline L-peak-systolic BFV & 120.0 & 101.4 & 0.009 \\
L-end-diastolic BFV & 49.3 & 38.1 & $<0.001$ \\
L-mean BFV & 77.3 & 59.6 & $<0.001$ \\
L-PI & 0.9 & 0.9 & 0.9 \\
L-RI & 0.58 & 0.59 & 0.3 \\
R-peak-systolic BFV & 120.8 & 95.8 & $<0.001$ \\
R-end-diastolic BFV & 49.7 & 37.1 & $<0.001$ \\
R-mean BFV & 77.1 & 58.7 & $<0.001$ \\
R-PI & 0.9 & 0.9 & 0.3 \\
R-RI & 0.57 & 0.58 & 0.9 \\
\hline
\end{tabular}

L, left; R, right; PI, pulsatility index; RI, resistance index; BFV; blood flow velocity.

coronary artery disease; age $>50$ years; hypothyroidism following thyroidectomy; and hypothyroidism in pregnancy or postpartum period were excluded. Consent was obtained from the local ethics committee, and all participants had signed informed consent forms.

\section{Transcranial Doppler examination}

Transcranial Doppler (TCD) ultrasonography examination was performed with Multi-Dop X DWL, TCD machine after the subjects rested in a supine position for $10 \mathrm{~min}$. The right and the left middle cerebral artery (MCA) were insonated from the temporal windows at 50-65 mm depth with $2 \mathrm{MHz}$ pulsed Doppler probes. Bilateral MCA peak-systolic, end-diastolic, and mean blood flow velocities; pulsatility index values, and resistance index values were recorded and then compared with each group. TCD was performed again when the patients with clinical hypothyroidism became euthyroid between 3 weeks and 3 months due to thyroid hormone replacement therapy (HRT). Before and after thyroid HRT, TCD findings were also compared in the clinical hypothyroid group.

\section{Statistical analysis}

The data was analyzed using the Statistical Package for Social Sciences (SPSS Inc, Chicago, IL, USA) 15.0 program. Paired $t$-test, one-way ANOVA, and Bonferroni analysis as post hoc test were used at statistical analysis. In all the analysis, $P$ value $<0.05$ was considered significant.

\section{Results}

This study comprised 30 clinical hypothyroid patients, 30 subclinical hypothyroid patients, and 30 healthy control subjects. Men/women ratio and mean age in clinical, subclinical hypothyroid, and control groups were $3 / 27,4 / 26$, and $5 / 25$ and $37.4,34.4$, and 36.7 respectively. Diagnosis was made in accordance with physical findings of patients and laboratory levels of their thyroid hormones. Fatigue, hoarseness, constipation, weight gain, hair loss, cold intolerance, and depression were most commonly seen in patients with clinical hypothyroidism in our study. Mean thyroid stimulating hormone (TSH) and free thyroxine $\left(\mathrm{T}_{4}\right)$ levels were $19.8 \mu \mathrm{IU} / \mathrm{ml}$ (range $0.4-4.0 \mu \mathrm{IU} / \mathrm{ml}$ ) and $0.5 \mathrm{ng} / \mathrm{dl}$ (range $0.8-1.9 \mathrm{ng} / \mathrm{dl}$ ), respectively, in clinical hypothyroid group. They all had autoimmune thyroiditis (also called Hashimoto's disease). Serum anti-thyroglobulin antibody was investigated in 27 of the 30 patients with clinical hypothyroidism and in 20 of the 30 patients with subclinical hypothyroidism. Their antibody results were all positive. Mean TSH and free $\mathrm{T}_{4}$ levels were $6.3 \mu / \mathrm{ml}$ (range $0.4-4.0 \mu / \mathrm{ml}$ ) and $1.2 \mathrm{ng} / \mathrm{dl}$ (range $0.8-1.9 \mathrm{ng} / \mathrm{dl}$ ), respectively, in subclinical hypothyroid group. This group had abnormal thyroid hormone levels without clinical symptom and sign.

TCD findings of clinical and subclinical hypothyroid groups compared with the control group are summarized in Tables 1 and 2. Peak-systolic, end-diastolic, and mean blood flow velocities were significantly higher in clinical and subclinical hypothyroid groups compared with control group $(P<0.05)$. However, peak-systolic, end-diastolic, and mean blood flow velocities were similar in clinical and subclinical hypothyroid groups $(P>0.05)$. In addition, the pulsatility index and resistance index values were not significantly different in all groups.

When the patients with clinical hypothyroidism became euthyroid after thyroid HRT, TCD was performed again, and the peak-systolic, end-diastolic, and mean blood flow velocities were significantly decreased after thyroid HRT in clinical hypothyroid group $(P<0.05$; Table 3$)$. However, the pulsatility index and resistance index values were not significantly different.

Table 2 Transcranial Doppler data of subclinical hypothyroid group compared with control group.

\begin{tabular}{lccc}
\hline & $\begin{array}{c}\text { Subclinical } \\
\text { hypothyroid }\end{array}$ & Control & $\boldsymbol{P}$ \\
\hline L-peak-systolic BFV & 121.3 & 101.4 & 0.005 \\
L-end-diastolic BFV & 49.6 & 38.1 & $<0.001$ \\
L-mean BFV & 78.5 & 59.6 & $<0.001$ \\
L-PI & 0.9 & 0.9 & 0.9 \\
L-RI & 0.5 & 0.59 & 0.9 \\
R-peak-systolic BFV & 125.0 & 95.8 & $<0.001$ \\
R-end-diastolic BFV & 51.9 & 37.1 & $<0.001$ \\
R-mean BFV & 80.0 & 58.7 & $<0.001$ \\
R-PI & 0.89 & 0.9 & 0.1 \\
R-RI & 0.5 & 0.58 & 0.8 \\
\hline
\end{tabular}

L, left; R, right; PI, pulsatility index; RI, resistance index; BFV; blood flow velocity. 
Table 3 Transcranial Doppler data of clinical hypothyroid group before and after thyroid hormone replacement therapy.

\begin{tabular}{lllc}
\hline & \multicolumn{3}{c}{ Clinical hypothyroid group } \\
\cline { 2 - 4 } & $\begin{array}{l}\text { Before } \\
\text { treatment }\end{array}$ & $\begin{array}{l}\text { After } \\
\text { treatment }\end{array}$ & $P$ \\
\hline L-peak-systolic BFV & 120.0 & 89.0 & $<0.001$ \\
L-end-diastolic BFV & 49.3 & 33.8 & $<0.001$ \\
L-mean BFV & 77.3 & 57.1 & $<0.001$ \\
L-PI & 0.9 & 0.9 & $>0.05$ \\
L-RI & 0.58 & 0.60 & $>0.05$ \\
R-peak-systolic BFV & 120.8 & 91.1 & $<0.001$ \\
R-end-diastolic BFV & 49.7 & 33.8 & $<0.001$ \\
R-mean BFV & 77.1 & 58.3 & $<0.001$ \\
R-PI & 0.9 & 0.9 & $>0.05$ \\
R-RI & 0.57 & 0.60 & $>0.05$ \\
\hline
\end{tabular}

L, left; R, right; PI, pulsatility index; RI, resistance index; BFV; blood flow velocity.

\section{Discussion}

The relationship with hypothyroidism and atherosclerosis was presented for the first time in an autopsy of myxedematous woman in 1878 (3). In 1967, Vanhaelst et al. (10) first made a case-control study and compared 25 autopsies with myxedema and 50 autopsies without myxedema. They found that coronary artery atherosclerosis was more common in the hypothyroid group (10). Following this, some investigators found that atherosclerosis in hypothyroidism was associated with hypercholesterolemia, hyperhomocysteinemia, increased C-reactive protein, hypertension, and impaired endothelial function $(2,3)$.

Nagasaki et al. (5) reported that carotid intima-media thickness was significantly higher in patients with clinical hypothyroidism than in euthyroid control subjects. Carotid intima-media thickness measured by ultrasonography represents early atherosclerotic changes and is generally accepted as a surrogate marker of future cardiovascular events $(5,11)$. Patients with hypothyroidism have been reported to have greater radial wall thickness and compliance than euthyroid healthy age- and sex-matched controls (12). Changes in the arterial wall, reducing elasticity, and increasing stiffness could induce increased systolic and diastolic blood pressure (3).

Thyroid hormones control blood pressure. In a study, it was found that hypertension increased threefold in patients with clinical hypothyroidism compared with the euthyroid group (13). Increased diastolic blood pressure was reported after thyroidectomy in normotensive persons (14). The potential mechanisms under this condition are increased peripheral vascular resistance and arterial stiffness $(9,15)$. Arterial stiffness is an important determinant of arteriosclerosis (16). Lekakis et al. (17) found that endothelium-associated vasodilatation was impaired in patients with hypothyroidism and they showed that it was improved by adequate thyroid HRT (18).
In this study, we investigated the effects of hypothyroidism on cerebral blood flow parameters. First, peaksystolic, end-diastolic and mean blood flow velocities and pulsatility index and resistance index values were similar in clinical and subclinical hypothyroid groups. However, the systolic, end-diastolic, and mean blood flow velocities were significantly higher in both clinical and subclinical hypothyroid groups than in the control group $(P<0.05)$. When patients with clinical hypothyroidism became euthyroid by thyroid HRT, the peaksystolic, end-diastolic, and mean blood flow velocities were significantly decreased $(P<0.05)$. In our study, TCD measurements were taken on proximal segments of right and left MCA. We did not observe a focal stenotic finding in any patients during TCD examination. The high MCA velocities on both hypothyroid groups may be a result of diffuse vessel abnormalities such as increased arterial stiffness or changes in systemic blood pressure. Vasomotor response to increased systemic blood pressure is an increase in cerebral blood flow velocity. It is reported that increased mild-to-moderate cerebral blood flow velocity is due to diffuse atherosclerosis or vasoconstriction secondary to systemic hypertension (19). In our study, the mechanisms that underlie significantly increased blood flow velocities of both the hypothyroid groups is most likely to be due to mild diffuse subclinical atherosclerosis and/or MCA vasoconstriction secondary systemic hypertension. Actually, we do not know the level of systemic blood pressure in both hypothyroid groups because we did not measure the patients' blood pressure during this study. Unfortunately, this may be a shortcoming of this study.

In our study, normalization of blood flow velocities in patients with clinical hypothyroidism was seen between 3-week and 3-month period with adequate thyroid HRT, suggesting that it is a reversible condition.

The pulsatility index and resistance index values were not significantly different in each group (Tables 1-3; $P>0.05)$. The pulsatility index and resistance index are arithmetic values that depends on vascular parameters. They are calculated as follows: pulsatility index $=$ (peak systolic velocity-end diastolic velocity)/mean blood flow velocity. Resistance index $=$ (peak systolic velocity-end diastolic velocity)/peak systolic velocity. These parameters reflect the flow velocity waveform and indicate the cerebrovascular resistance $(20,21)$. Pulsatility index and resistance index may change with proximal or distal occlusion, high intracranial pressure, and decreased compliance in older age. Kwater et al. (22) pointed a relationship between pulsatility index and resistance index and systemic arterial stiffness in atherosclerotic population. A normal pulsatility index and resistance index value in our study may be explained by mild or early phase of vascular changes due to hypothyroidism. There are also some SPECT studies on hypothyroidism in which demonstrated reversible regional cerebral blood flow changes but 
cerebral blood flow does not change with cerebral blood velocity (23).

In conclusion, increased cerebral blood flow velocities were seen in patients with clinical and subclinical hypothyroidism. Normalization of increased blood flow velocities with adequate thyroid HRT can suggest a reversible condition.

\section{Declaration of interest}

The authors declare that there is no conflict of interest that could be perceived as prejudicing the impartiality of the research reported.

\section{Funding}

This research did not receive any specific grant from any funding agency in the public, commercial or not-for-profit sector.

\section{References}

1 Klein I \& Ojamaa K. Thyroid hormone and the cardiovascular system. New England Journal of Medicine 2001244 501-507.

2 Cappola AR \& Ladenson PW. Hypothyroidism and atherosclerosis. Journal of Clinical Endocrinology and Metabolism $2003 \mathbf{8 8}$ 2438-2444. (doi:10.1210/jc.2003-030398)

3 Ichiki T. Thyroid hormone and atherosclerosis. Vascular Pharmacology 201052 151-156. (doi:10.1016/j.vph.2009.09.004)

4 Hak AE, Pols HAP, Visser TJ, Drexhage HA, Hofman A \& Witteman JCM. Subclinical hypothyroidism is an independent risk factor for atherosclerosis and myocardial infarction in elderly women: the Rotterdam study. Annals of Internal Medicine 2000 132 270-278.

5 Nagasaki T, Inaba M, Henmi Y, Kumeda Y, Ueda M, Tahara H, Sugiguchi S, Fujiwara S, Emoto M, Ishumura E, Onoda N, Ishikawa $\mathrm{T} \&$ Nishizawa Y. Decrease in carotid intima-media thickness in hypothyroid patients after normalization of thyroid function. Clinical Endocrinology 200359 607-612. (doi:10.1046/ j.1365-2265.2003.01893.x)

6 Sun MY, Chen TC \& Lee YL. Hypothyroidism and cerebral infarction: a case report and literature review. Acta Neurologica Taiwanica 200615 197-200.

7 Blois SL, Poma R, Stalker MJ \& Allen DG. A case of primary hypothyroidism causing central nervous system atherosclerosis in a dog. Canadian Veterinary Journal 200849 789-792.

8 Ojamaa K, Klemperer JD \& Klein I. Acute effects of thyroid hormone on vascular smooth muscle. Thyroid $1996 \mathbf{6} 505-512$. (doi:10.1089/thy.1996.6.505)

9 Obuobie K, Smith J, Evans M, John R, Davies JS \& Lazarus JH. Increased central arterial stiffness in hypothyroidism. Journal of Clinical Endocrinology and Metabolism $2002874662-4666$. (doi:10.1210/jc.2002-020493)

10 Vanhaelst L, Neve P, Chailly P \& Bastenie PA. Coronary artery disease in hypothyroidism. Observations in clinical myxoedema. Lancet 19672 800-802. (doi:10.1016/S01406736(67)92235-0)
11 O'Leary DH \& Polak JF. Intima-median thickness: a tool for atherosclerosis imaging and event prediction. American Journal of Cardiology 200290 18-21. (doi:10.1016/S0002-9149 (02)02957-0)

12 Giannattasio C, Rivolta MR, Failla M, Mangonif AA, Stellaf ML \& Mancia G. Large and medium sized artery abnormalities in untreated and treated hypothyroidism. European Heart Journal $1997181492-1498$.

13 Saito I \& Saruta T. Hypertension in thyroid disorders. Endocrinology and Metabolism Clinics of North America 199423 379-386.

14 Fommei E \& Iervasi G. The role of thyroid hormone in blood pressure homeostasis: evidence from short-term hypothyroidism in humans. Journal of Clinical Endocrinology and Metabolism 2002 87 1996-2000. (doi:10.1210/jc.87.5.1996)

15 Graettinger JS, Muenster JJ, Checchia CS, Grisson RL \& Campbell JA. A correlation of clinical and hemodynamic studies in patients with hypothyroidism. Journal of Clinical Investigation 19589 502-510. (doi:10.1172/JCI103631)

16 Mattace-Raso FUS, van der Cammen TJM, Hofman A, van Popele NM, Bos ML, Schalekamp MA, Asmar R, Reneman RS, Hoeks AP, Breteler MM \& Witteman JC. Arterial stiffness and risk of coronary heart disease and stroke: the Rotterdam study. Circulation 2006113 657-663. (doi:10.1161/CIRCULATIONAHA.105.555235)

17 Lekakis J, Papamichael C, Alevizaki M, Piperingos G, Marafelia P, Mantzos J, Stamatelopoulos S \& Koutras DA. Flow-mediated, endothelium-dependent vasodilation is impaired in subjects with hypothyroidism, borderline hypothyroidism, and high-normal serum thyrotropin (TSH) values. Thyroid $1997 \quad \mathbf{7} 411-413$. (doi:10.1089/thy.1997.7.411)

18 Taddei S, Caraccio N, Virdis A, Dardano A, Versari D, Ghiadoni L, Salvetti A, Ferrannini E \& Monzani F. Impaired endotheliumdependent vasodilatation in subclinical hypothyroidism: beneficial effect of levothyroxine therapy. Journal of Clinical Endocrinology and Metabolism $2003 \mathbf{8 8}$ 3731-3737. (doi:10.1210/jc.2003030039)

19 Bos MJ, Koudstaal PJ, Hofman A, Witteman JCM \& Breteler MMB. Transcranial Doppler hemodynamic parameters and risk of stroke. The Rotterdam study. Stroke 200738 2453-2458. (doi:10.1161/ STROKEAHA.107.483073)

20 Gosling RG \& King DH. Arterial assessment by Doppler shift ultrasound. Proceedings of the Royal Society of Medicine $1974 \mathbf{6 7}$ 447-449.

21 Pourcelot L. Diagnostic ultrasound for cerebrovascular disease. In Present and Future of Diagnostic Ultrasound, pp 141-147. Ed. I Donald, Rotterdam, The Netherlands: Kooyker, 1976.

22 Kwater A, Gsowski J, Gryglewska B, Wizner B \& Grodzicki T. Is blood flow in the middle cerebral artery determined by systemic arterial stiffness? Blood Pressure 200918 130-134. (doi:10.1080/ 08037050902975114)

23 Nagamachi S, Jinnouchi S, Nishii R, Ishida Y, Fujita S, Futami S, Kodama T, Tamura S \& Kawai K. Cerebral blood flow abnormalities induced by transient hypothyroidism after thyroidectomy analysis by tc-99m-HMPAO and SPM96. Annals of Nuclear Medicine 200418 469-477. (doi:10.1007/BF02984562)

Received 19 March 2011

Revised version received 29 June 2011

Accepted 6 July 2011 